\title{
BMJ Open Retrospective cohort analysis of prescription patterns of cancer medications during periods of drug stockouts in Botswana
}

\author{
Sonya Davey (D , ${ }^{1}$ Surbhi Grover, ${ }^{2}$ Warren B Bilker, ${ }^{3}$ Dipho I Setlhako, ${ }^{4}$ \\ Tlotlo B Ralefala, ${ }^{4}$ Patrick Manshimba, ${ }^{4}$ Robert Gross, ${ }^{3,5}$ Angela DeMichele, ${ }^{3,6}$ \\ Lawrence N Shulman, ${ }^{6}$ Yehoda M Martei (i) ${ }^{6}$
}

To cite: Davey S, Grover S, Bilker WB, et al. Retrospective cohort analysis of prescription patterns of cancer medications during periods of drug stockouts in Botswana. BMJ Open 2021;11:e049574. doi:10.1136/ bmjopen-2021-049574

- Prepublication history for this paper is available online To view these files, please visit the journal online (http://dx.doi. org/10.1136/bmjopen-2021 049574).

Received 27 January 2021 Accepted 23 June 2021

\section{Check for updates}

(C) Author(s) (or their employer(s)) 2021. Re-use permitted under CC BY-NC. No commercial re-use. See rights and permissions. Published by BMJ.

For numbered affiliations see end of article.

Correspondence to Dr Yehoda M Martei; yehoda.martei@ennmedicine. upenn.edu

\section{ABSTRACT}

Objective Cancer drug stockouts occur at high frequencies globally, however, their effects on treatment are understudied in sub-Saharan Africa (SSA). We aimed to determine whether causes of suboptimal cancer treatment prescriptions differed between periods of stockout and full treatment supply.

Design A retrospective cohort study of systemic therapy prescriptions for patients diagnosed with the twelve most common solid tumour cancers treated in 2016.

Setting Princess Marina Hospital in Gaborone, Botswana. Participants Patients in the retrospective cohort who experienced any suboptimal treatment events, defined as $\geq 7$ days delay or switch from guideline-concordant initiated therapy.

Primary and secondary outcome measures Frequency of delays and patterns of prescription changes for specific regimens and cancer types.

Results $167 / 378$ patients contributed to 320 suboptimal events (115 therapy switches, 167 delays and 38 events with both), over 1452 total chemotherapy cycles received. Events during stockout were $43 \%$ delays, $43 \%$ switches and $14 \%$ both during stockout periods and $67.2 \%$ delays, $24.4 \%$ switches and $8.4 \%$ both during non-stockout periods $(p<0.001)$. Majority of switches involved deescalation of initially prescribed guideline-recommended regimens in patients with breast cancer, Kaposi sarcoma and patients with colorectal cancer, which occurred more frequently during periods of drug stockouts. Among patients with breast cancer, substitution of docetaxel for paclitaxel event occurred exclusively during paclitaxel drug stockout. Delays of $\geq 7$ days events were most frequent in breast cancer patients receiving paclitaxel during stockout, and combination doxorubicin and cyclophosphamide even during periods of non-stockout.

Conclusions The aetiology of suboptimal events differed during stockout and non-stockout periods. Prescription patterns that involved de-escalation of initiated therapy and substitution of paclitaxel with docetaxel occurred frequently during periods of drug stockout. Further research needs to be conducted to understand the impact of stockout on survival and barriers to maintaining essential cancer medicines supplies in SSA, and the factors driving frequent delays in therapy delivery.
Strengths and limitations of this study

- Retrospective analysis of all patients with the twelve most common solid tumours treated with chemotherapy at Princess Marina Hospital in 2016 leading to an unbiased population sample.

- Rigorous measurement of stockout exposure and strict criteria for ascertainment of suboptimal events including delay and regimen switch.

- Does not include patient outcome data.

- No data on reasons for physicians' decision making for switches and delays during stockout and nonstockout periods.

\section{INTRODUCTION}

As the global cancer burden increases, more cancer medicines have been added to the WHO Model Lists of Essential Medicines (EML) and subsequently national medicines lists, including that of Botswana, to promote and expand access to cancer drugs globally. ${ }^{12}$ However, there are significant challenges with ensuring reliable medicine supply, especially in low-income and middle-income countries. An analysis of the alignment of national EML of countries in sub-Saharan Africa (SSA) compared with the WHO EML showed that a median number of 14 cancer drugs appeared on national essential medicines list (NEML) out of 25 cancer medicines in 2013 and 16 added in $2015 .{ }^{3}{ }^{4}$ Furthermore, even when medicines are listed on the NEML, it does not guarantee availability at the point of care for patients. ${ }^{56}$

The WHO's working group definition of stockout is 'the complete absence of the medicine, health product or vaccine at the point of service delivery to the patient, ${ }^{7}$ while medicine shortage is defined as "the supply of medicines, health products and vaccines identified as essential by the health system 
is considered to be insufficient to meet public health and patient needs. ${ }^{7}$ In prior studies, anticancer medicine shortage in high-income countries have resulted in suboptimal therapy delivery because of drug substitutions that are less effective, more toxic and/or resulted in treatment delays. ${ }^{8-11}$ Suboptimal therapy delivery leads to reduced chemotherapy relative dose intensity, which leads to inferior disease free and overall survival outcomes. ${ }^{11-15}$

There is a critical gap in our knowledge of the role of drug stockouts in oncology treatment in SSA countries, such as Botswana. ${ }^{16} 17$ These data are important for understanding the clinical impact of stockouts on patient care, in order to inform future design of targeted interventions to improve cancer therapy delivery. In Botswana, the Central Medical Store (CMS) is a semiautonomous public sector agency that procures and distributes drugs and other healthcare commodities to health facilities, including clinics and hospitals, throughout the country. ${ }^{5}$ We have previously shown that the Botswana NEML has one of the highest alignments with the WHO EML for cancer medicines in SSA, however, $40 \%$ of the drugs on the Botswana NEML were out of stock for a median of a month in previous years, ${ }^{5}$ which doubled the risk of suboptimal therapy delivery. ${ }^{17}$

The objective of this analysis is to determine whether the causes of suboptimal treatment course differed between periods of stockout and full treatment supply by assessing prescription patterns of systemic chemotherapy in cancer patients in Botswana whose regimens did not meet standard guidelines defined by either switches or delays in therapy. Medicines included in the analysis are currently listed on the WHO EML and the Botswana NEML.

\section{METHODS}

\section{Study design and participants}

The present analysis examines only patients with at least one suboptimal therapy event in our retrospective cohort study of patients diagnosed with the most common solid tumour malignancies who received systemic chemotherapy at Princess Marina Hospital (PMH), Gaborone, Botswana in 2016. ${ }^{17}$ The overall study population consisted of 378 patients, age $\geq 18$ years, with a diagnosis of any of the following 12 cancers-Kaposi sarcoma (KS), anal, breast, cervical, colon, oesophageal, head and neck, lung, nasopharyngeal, ovarian, prostate, rectal or uterine cancers who received at least one cycle of systemic treatment from 1 January 2016 to 31 December 2016. Patients with haematological malignancies were excluded because of variability in care referrals-a proportion of patients are referred to South Africa, India and private hospitals within Botswana for specific expertise and multiple lines of treatment when indicated. The study site was PMH because the hospital provides the highest volume of cancer care in Botswana.

\section{Drug stockout measures}

The presence of drug stockout was defined as a stockout of $\geq 1$ day(s) of any drug component of the patient's intended regimen occurring within a cycle interval. The stockout duration was computed as the first date the drug was reported as being out of stock by CMS to the date the drug was reported as being back in stock. The duration of a drug stockout occurring during a cycle interval for any of the drugs prescribed during the respective cycles was calculated as the days of stockout between cycles, for example, cycle 1 day 1 and cycle 2 day 1 (figure 1 ). If more than one drug stockout occurred during a cycle, the higher number of stockout days was assigned to total stockout days for the cycle. Duration of stockout and association with treatment cycle for each patient was generated via six possible patterns of stockout: (1) stockout occurred during cycle interval, (2) stockout started before cycle 1 but continued during cycle interval, (3) stockout started during cycle interval and extended into cycle 2, (4) stockout occurred prior to cycle 1, (5) stockout occurred after cycle 2 and (6) stockout started prior to cycle 1 and extended beyond cycle 2 (figure 1 ). In any permutation in which the stockout occurred prior to, or after a specific cycle interval, we were unable to know whether therapy was initiated on time vs therapy was delayed. Therefore, patterns 4 and 5 were excluded from the analysis respective to events occurring between cycle 1 and cycle 2. Permutations in which cycle 2 of the 'stockout drug' was administered (pattern 3 and 6), although there a stockout reported by CMS, may represent an external supply of medicines outside of CMS, for example, outof-pocket purchase by the patient. If a stockout occurred after cycle 2, then it was not considered an exposure during cycle 2 because the stockout occurred after the cycle (pattern 5). Each cycle was considered a separate event. Using STATA (STATA), the number of days of drug stockout per specific cycle interval was calculated.

\section{Outcome measures}

Three units of analyses are incorporated in our analysespatient level, event level and the total number of cycles received. Each patient may have multiple events and multiple cycles based on the treatment regimen prescribed. Statistical analysis for the different level of analyses is discussed below. We included only patients who had at least one suboptimal therapy delivery event during their treatment course, defined as $\geq 7$ days delay, missed cycle or switch in initially prescribed standard guideline regimen. These parameters were used to define suboptimal therapy event because any of these events can result in reduced relative dose intensity of therapy which has been associated with inferior cancer specific and overall survival. ${ }^{18}{ }^{19}$ Suboptimal therapy events are analysed per event. We then evaluated the proportion of these that occurred during periods dichotomised into stockout versus drugs being in adequate supply (non-stockout). Standard guideline regimen was determined using the National Comprehensive Cancer Network (NCCN) Clinical Practice Guidelines in Oncology ${ }^{20}$ and 


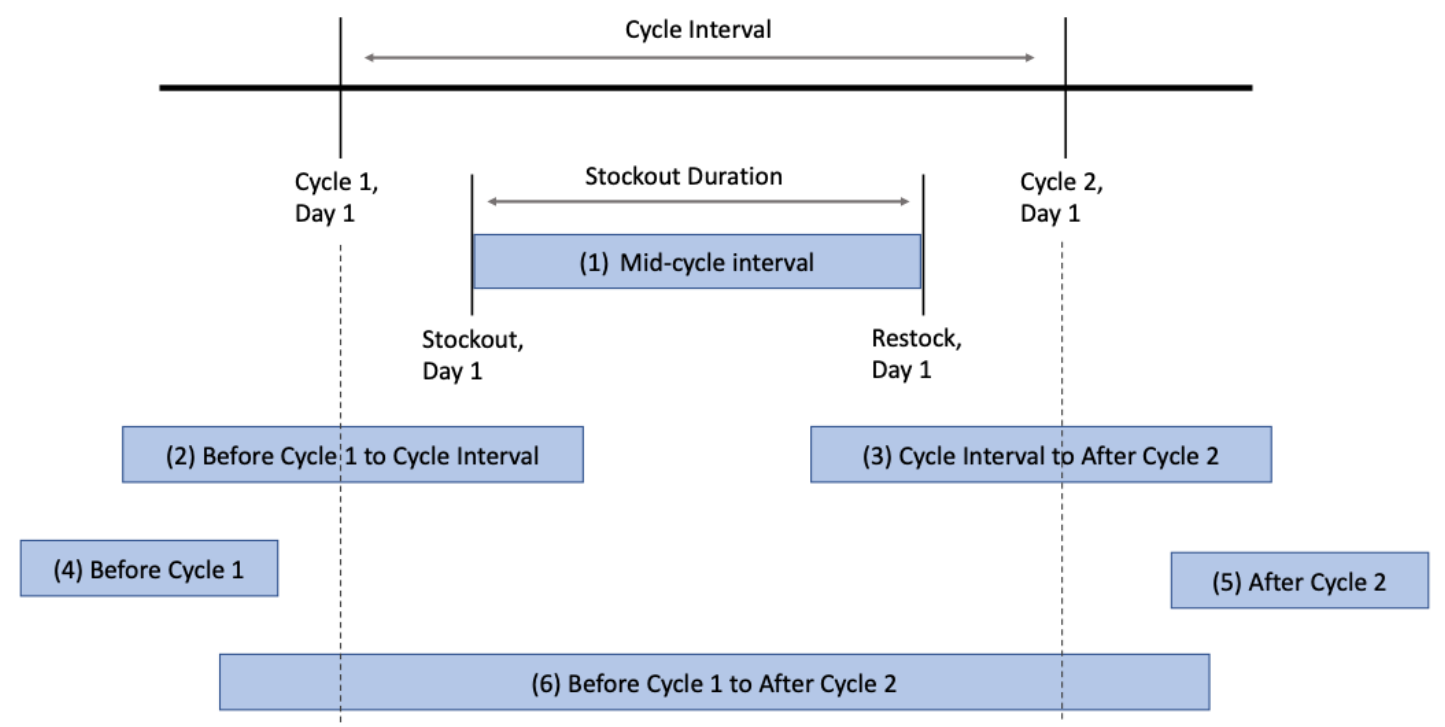

Figure 1 Duration of stockout during each treatment cycle for each patient was generated via six possible patterns of stockout association with cycle interval: (1) stockout occurred during cycle interval, (2) stockout started before cycle one but continuing during cycle interval, (3) stockout started during cycle interval and extended into cycle 2, (4) stockout occurred prior to cycle 1, (5) stockout occurred after cycle 2, (6) stockout started during cycle one and extended into cycle 2. Adapted from Martei et al. ${ }^{17}$

the WHO supplemental guidelines for the WHO EML. ${ }^{1}$ Our definition of suboptimal therapy events assumes that the original therapy initiated was the optimal therapy based on patient and disease characteristics and provider's clinical reasoning, however, we are unable to verify this with specific data based on the study design of our retrospective analysis.

\section{Statistical analysis}

$\chi^{2}$ or Fisher's exact tests (when expected cell size was $<5$ ) were used to compare dichotomous patient baseline characteristics, and Student's t-test were used to compare continuous variables between stockout versus non-stockout periods on a patient-level. $\chi^{2}$ test or Fisher's exact tests (when expected cell size was $<5$ ) were used to compare the proportion of switches and delays occurring during periods of stockout vs non-stockout using an event-level analyses. The Wilcoxon rank sum tests were used to compare median delays (if delay was $\geq 7$ days) by stockout versus non-stockout. The frequency of cycles with discrete prescription patterns that deviated from guideline-initiated therapy were computed including: $\geq 7$ days delay in cycle length and switch from guideline-concordant initiated therapy. We then compared the proportion of those patterns present during periods of drug stockout versus non-stockout periods. The analysis was limited to prescription patterns that occurred more than once during the study period. All analyses were performed in STATA V.16.

\section{Patient and public involvement}

It was not possible to involve patients or the public in the design, or conduct, or reporting, or dissemination plans of our research.

\section{RESULTS}

Of the 378 patients with one of the ten most common solid tumour malignancies who received systemic cancer therapy at PMH in 2016, $167(44.2 \%)$ patients experienced at least one suboptimal treatment course.

A summary of baseline characteristics of the 167 patients are presented in table 1 using a patient-level analysis of the data. A significant majority of the patients included in the analysis were female ( $68.9 \%$ vs $24 \%$ male, $p=0.012$ ) and the mean age for patients who experienced a drug stockout during their treatment was significantly higher, 51.9 , vs 47.6 years for patients who did not experience a drug stockout during their treatment course $(p=0.044)$. Breast cancer was the most common cancer diagnosis $(54.5 \%)$ during both stockout and non-stockout periods. There were no significant differences in the distribution of HIV status, stage, cancer diagnosis and intent of guideline-initiated therapy (table 1 ).

Of the 320 total suboptimal events, the majority of events during non-stockout were delays. table 2 provides a detailed breakdown of event-level analysis which showed a significant difference in the distribution of events during stockout compared with non-stockout. There was no significant difference in the median duration of delay during periods of stockout (17 days, IQR 11-29) vs nonstockout (14.5 days, IQR 11-22) in those with suboptimal courses $(\mathrm{p}=0.16)$.

Figure 2 shows the frequency of specific prescription patterns that represented a switch from guideline-initiated therapy to an alternative regimen based on event-level analysis. Breast cancer patients experienced the highest 
Table 1 Baseline characteristics of patients who had at least one suboptimal therapy delivery event stratified by whether there were drug stockouts during their treatment plan versus not

\begin{tabular}{|c|c|c|c|c|}
\hline Patient characteristic & $\begin{array}{l}\text { Periods of drug } \\
\text { stockout ( } N=113)\end{array}$ & $\begin{array}{l}\text { Periods of no drug } \\
\text { stockout ( } N=54)\end{array}$ & Total $(\mathrm{N}=167)$ & $P$ value \\
\hline Mean age $(95 \% \mathrm{Cl})$ & 51.9 (49.4 to 54.3$)$ & 47.6 (44.3 to 50.9$)$ & 50.5 (48.5 to 52.4$)$ & 0.044 \\
\hline Missing & 2 & 1 & 3 & \\
\hline \multicolumn{5}{|l|}{ Sex } \\
\hline Female & $85(75 \%)$ & $30(56 \%)$ & 115 (68.9\%) & 0.012 \\
\hline Male & $21(19 \%)$ & 19 (35\%) & 40 (24.0\%) & \\
\hline Missing & $7(6 \%)$ & $5(9 \%)$ & $12(7.1 \%)$ & \\
\hline \multicolumn{5}{|l|}{ HIV status } \\
\hline HIV-positive & $30(27 \%)$ & $18(33 \%)$ & $48(28.7 \%)$ & 0.98 \\
\hline HIV-negative & $32(28 \%)$ & 19 (35\%) & $51(30.5 \%)$ & \\
\hline Missing & $51(45 \%)$ & $17(31 \%)$ & $68(40.8 \%)$ & \\
\hline \multicolumn{5}{|l|}{ Stage } \\
\hline Stage 1 & $1(1 \%)$ & $0(0.0 \%)$ & $1(0.6 \%)$ & 0.897 \\
\hline Stage 2 & $14(12 \%)$ & $4(7 \%)$ & $18(10.8 \%)$ & \\
\hline Stage 3 & $32(28 \%)$ & $11(20 \%)$ & 43 (25.7\%) & \\
\hline Stage 4 & $20(18 \%)$ & $9(17 \%)$ & $29(17.4 \%)$ & \\
\hline Missing & $46(41 \%)$ & $30(56 \%)$ & $76(45.5 \%)$ & \\
\hline \multicolumn{5}{|l|}{ Cancer diagnosis } \\
\hline Anal cancer & $1(1 \%)$ & $2(4 \%)$ & $3(1.8 \%)$ & 0.117 \\
\hline Breast cancer & $68(60 \%)$ & $23(43 \%)$ & $91(54.5 \%)$ & \\
\hline Cervical cancer & $2(2 \%)$ & $1(2 \%)$ & $3(1.8 \%)$ & \\
\hline Colon cancer & $8(7 \%)$ & $6(11 \%)$ & $14(8.4 \%)$ & \\
\hline Head \& neck cancer & $4(4 \%)$ & $1(2 \%)$ & $5(3.0 \%)$ & \\
\hline Kaposi sarcoma & $14(12 \%)$ & $17(31 \%)$ & $31(18.6 \%)$ & \\
\hline Lung cancer & $2(2 \%)$ & $0(0 \%)$ & $2(1.2 \%)$ & \\
\hline Nasopharyngeal cancer & $2(2 \%)$ & $0(0 \%)$ & $2(1.2 \%)$ & \\
\hline Ovarian cancer & $4(4 \%)$ & $3(6 \%)$ & $7(4.2 \%)$ & \\
\hline Prostate cancer & $2(2 \%)$ & $0(0 \%)$ & $2(1.2 \%)$ & \\
\hline Rectal cancer & $4(4 \%)$ & $1(2 \%)$ & $5(3.0 \%)$ & \\
\hline Uterine cancer & $2(2 \%)$ & $0(0 \%)$ & $2(1.2 \%)$ & \\
\hline \multicolumn{5}{|l|}{ Intent of treatment regimen } \\
\hline Curative & $56(50 \%)$ & $20(37 \%)$ & $76(45.5 \%)$ & 0.062 \\
\hline Palliative & $32(28 \%)$ & $23(43 \%)$ & 55 (32.9\%) & \\
\hline Missing & $25(22 \%)$ & $11(20 \%)$ & $36(21.6 \%)$ & \\
\hline
\end{tabular}

frequency of therapy switches with most occurring during periods of drug stockout. The most common therapy switches across the disease groups involved de-escalation of guideline-concordant initiated therapy. For patients with breast cancer, this included de-escalation from paclitaxel and trastuzumab combination for patients with

Table 2 Event-level analysis of distribution of delays and switches during periods of drug stockout compared with periods of non-stockout

\begin{tabular}{lllll}
\hline Event-type & Periods of drug stockout $\mathbf{( n}(\%))$ & Periods of non-stockout $\mathbf{( n}(\%))$ & Total N (\%) & P value \\
\hline Delays & $87(43)$ & $80(67.2)$ & $167(52)$ & $<0.001$ \\
Switches & $86(43)$ & $29(24.4)$ & $115(36)$ & \\
Both & $28(14)$ & $10(8.4)$ & $38(12)$ & \\
Total & 201 & 119 & 320 & \\
\hline
\end{tabular}


Comparative frequency of therapy switches occuring during periods of stockout vs no drug stockout

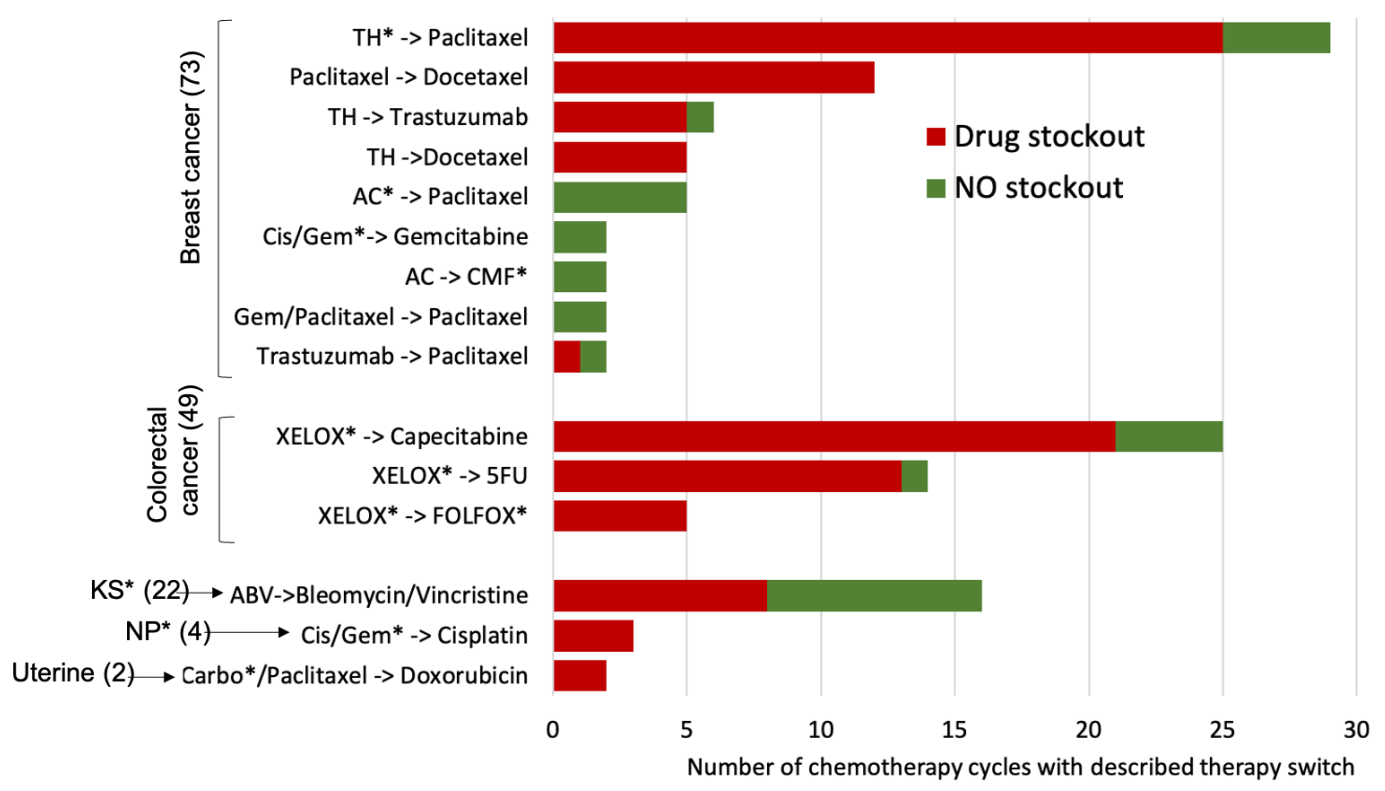

Figure 2 Comparative frequency of therapy switches occurring during periods of drug stockout versus periods when full stock of drugs is available. The respective diagnoses for which the regimen was prescribed are listed on the left. The respective total number of cycles for each diagnosis in our cohort is included in parenthesis. Bar graphs show patterns occurring more than once. *Describes switch from preceding regimen to following regimen. 5FU, fluorouracil; ABV, doxorubicin, bleomycin, vincristine; AC, doxorubicin and cyclophosphamide; carbo, carboplatin; Cis/Gem, cisplatin and gemcitabine; CMF, cyclophosphamide, methotrexate, fluorouracil; FOLFOX, fluorouracil, oxaliplatin, leucovorin; KS, Kaposi Sarcoma; NP, nasopharyngeal cancer; $\mathrm{TH}$, paclitaxel and trastuzumab; XELOX, capecitabine and oxaliplatin.

HER2 positive breast cancer to paclitaxel alone. Patients with colorectal cancer also had cycles during which there was de-escalation of therapy from capecitabine and oxaliplatin combination to capecitabine or intravenous fluorouracil only. For patients receiving treatment for KS, there was a switch from a combination of doxorubicin, bleomycin and vincristine to bleomycin and vincristine only. For patients with KS, this occurred equally during periods of drug stockout and non-stockout periods (figure 2). The second most frequent therapy switch for breast cancer patients was a within class switch from paclitaxel to docetaxel which occurred exclusively during periods of drug stockout (figure 2). Additional prescription behaviours are shown for other disease groups in figure 2.

Delays in cycle length of 7 or more days were more common in regimens prescribed for patients with breast cancer (figure 3). The regimens that experienced the most frequent delays were during cycles when patients received paclitaxel or when patients received a combination of doxorubicin and cyclophosphamide. Events corresponding to paclitaxel delays occurred more frequently during periods of stockout, whereas delays in cycles where a combination of doxorubicin and cyclophosphamide were prescribed occurred more frequently during nonstockout periods. Delays in administering combination of doxorubicin, bleomycin and vincristine for patients with KS occurred more frequently during periods of stockout (figure 3). For patients with colorectal cancer, delays during therapy was frequent for patients prescribed a combination of capecitabine and oxaliplatin and were more likely to occur during periods drug stockout. Additional data on cycle length delays for other disease groups and regimens are shown in figure 3 .

\section{DISCUSSION}

This manuscript provides a detailed comparative analysis of changes in prescription patterns that occur during periods of drug stockout. The analysis showed a significant difference in the distribution of suboptimal events during stockout versus non-stockout. The majority of suboptimal events occurring during non-stockout periods were delays and therapy switches and delays occurred with similar frequency during periods of stockout.

Consistent with our prior analysis, patients with breast cancer and therefore women, given the collinearity between breast cancer diagnosis and the female sex contributed the largest frequency of administered cycles in this analysis. Among breast patients, frequent prescription patterns included a de-escalation of therapy for patients with HER2-positive breast cancer. Additionally, patients with breast cancer receiving paclitaxel were switched to docetaxel during periods of paclitaxel stockout. De-escalation of systemic therapy for HER2 positive patients either in the curative or palliative setting is concerning for inferior therapy in a subset of patients for whom large clinical trials have shown that the addition of 


\section{Comparative frequency of $\geq 7$-day delay in cycle length occuring during periods of stockout vs no drug stockout}

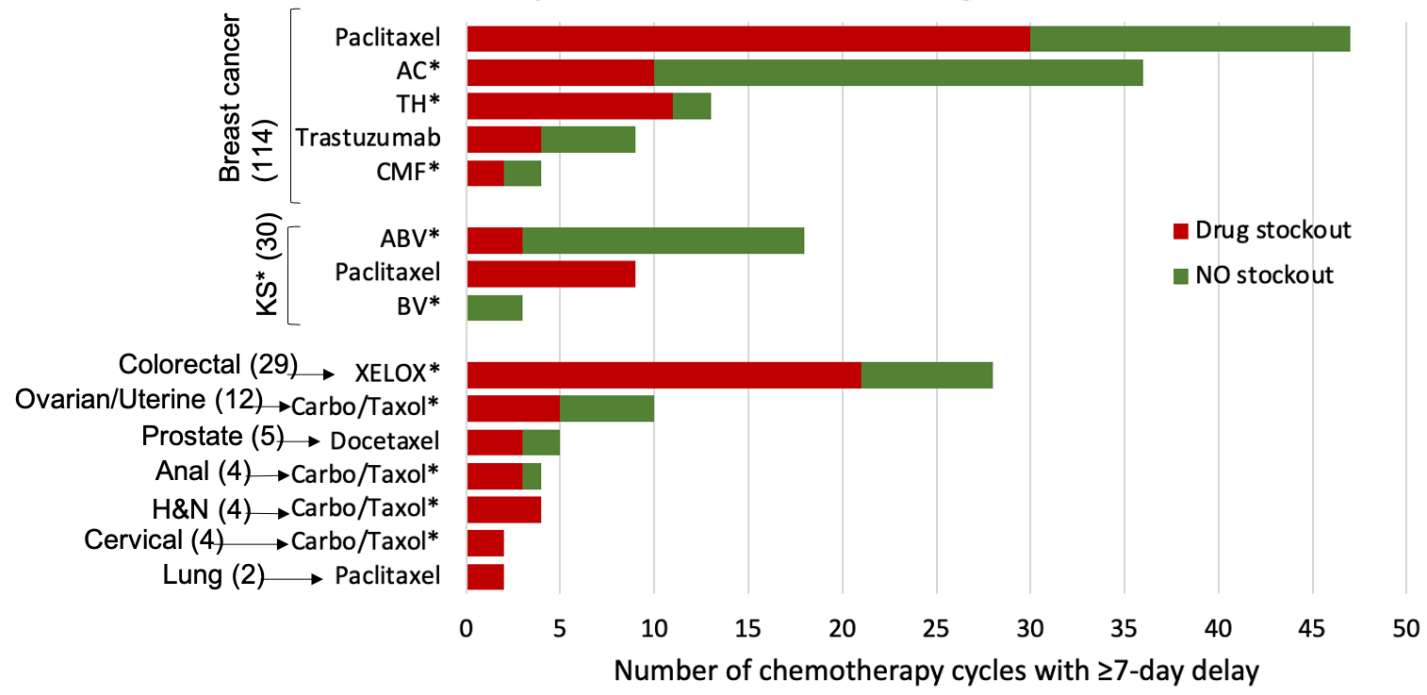

Figure 3 Comparative frequency of $\geq 7$ days delay in cycle length occurring during periods of drug stockout versus periods when full stock of drugs is available. The respective diagnoses for which the regimen was prescribed are listed on the left. The respective total number of cycles for each diagnosis in our cohort is included in parenthesis. Bar graphs show patterns occurring more than once. *Diagnosis and regimen key: ABV, doxorubicin, bleomycin, vincristine; AC, doxorubicin and cyclophosphamide; BV, bleomycin, vincristine; Carbo/Taxol, carboplatin and paclitaxel; CMF, cyclophosphamide, methotrexate, fluorouracil; H\&N, head and neck cancer (not including nasopharyngeal cancer); KS, Kaposi Sarcoma; TH, paclitaxel and trastuzumab; XELOX, capecitabine and oxaliplatin.

trastuzumab to paclitaxel results in superior overall and disease-free survival in HER2+ patients. $^{21}{ }^{22}$ In patients who were switched from paclitaxel to docetaxel, this switch may be equally efficacious with regards to survival outcomes, and in fact docetaxel is commonly prescribed as first line as paclitaxel in several institutions. ${ }^{23}$ It is noteworthy, however, that although equally efficacious it may be associated with higher rates of toxicity ${ }^{23}$ and higher costs. $^{24}$

Patients with colon and rectal cancers were also at a high risk of a suboptimal event due to regimen stockout. ${ }^{17}$ In this instance, capecitabine and oxaliplatin combinations were most commonly substituted with capecitabine or fluorouracil monotherapy during periods of oxaliplatin stockout. In patients with stage III and high-risk disease being treated with curative intent this switch may result in inferior overall and disease-specific survival. ${ }^{25} 26$ In patients with stage IV disease being treated with palliative intent, the clinical impact of such a switch in therapy is less certain, and in some age groups may be appropriate without compromising efficacy. Switch in therapy from capecitabine to fluorouracil are equally efficacious, however, the costs of administration may be higher in the Botswana setting where there are limited inpatient beds and patients receiving 48-hour infusion have to be admitted overnight in the hospital to complete their infusion therapy.

The results were also notable for a high frequency of at least $\geq 7$ days delays that occurred non-differentially during periods of stockout and periods of non-stockout. Frequent delays in therapy, especially in a low-income and middle-income setting where most patients are diagnosed with locally advanced disease may lead to inferior survival resulting from reduced dose intensity of curative therapy. ${ }^{27}$ This distribution suggests that delays are less likely related to medicines stock and may be associated with patient-related factors. Reasons for this high proportion of delays has not been well studied in the context of therapy delivery in SSA. We postulate that some reasons might include grade 3 or 4 toxicity requiring therapy delays, especially when growth factor support is not administered prophylactically, for example, in patients with breast cancer receiving a combination of doxorubicin and cyclophosphamide followed by paclitaxel as is recommended in USA and European guidelines. $^{28} 29$ Other reasons may include distance from the hospital and cost of transportation. ${ }^{30}$ Alternatively, it is possible that providers may switch therapies to a different regimen if they anticipate a prolonged drug stockout. In a prior analysis, we showed a median stockout duration of 1 month. ${ }^{5}$ The high frequency of delays is an area that warrants further studies to identify patient and provider factors that contribute to therapy delay and how this impacts clinical outcomes.

Our analysis had several limitations. We analysed changes in prescription patterns that occur during periods of stockout, which does not necessarily convey the clinical impact on patient survival outcomes. However, robust randomised clinical trial data provide support evidence that some, although not all of these switches are inferior. Long-term follow-up is needed to assess which of these switch events impact patient outcomes. 
Second, our outcome of interest, which examined specific prescription pattern switches and delays, may have been appropriate in patients with adverse events, irrespective of stockout. Our retrospective analysis did not include data on reasons for physicians' decision making which limits our assessment of whether delays and switches were intentional or not (eg, due to stockouts vs clinical indication vs other patient factors). In our analysis, few of these switches may have been appropriate and consistent with standard of care based on patient and clinical parameters at the time of the treatment recommendation. For instance, the NCCN guidelines note that combination therapy may be appropriate for patients with breast cancer presenting with a visceral crisis. Therefore, a de-escalation from gemcitabine-combination therapy to monotherapy in our analysis (figure 2) may have been appropriate in both scenarios and not a suboptimal event, but we are unable to assess this in our retrospective analysis. To our knowledge, there are no current studies or surveys evaluating the decision-making process of cancer care providers in SSA. However, it is plausible that therapy switches are more likely due to stockout. Surveys of physicians and pharmacists conducted in the USA on the impact of oncology drug shortages found that $93 \%$ of drug shortages led to regimen changes and $85 \%$ led to increased costs, ${ }^{31} 30 \%$ of substituted regimens during drug shortages were inferior and $35 \%$ were more toxic. ${ }^{9}$

Finally, the data only reflects patients getting systemic therapy at PMH, and therefore, do not reflect the total incidence of cancer cases diagnosed and treated in Botswana. Cervical cancer is the most commonly diagnosed cancer among women in Botswana, however, majority of patients are referred to a private health facility for definitive treatment with concurrent chemotherapy and radiation therapy and are therefore underrepresented in our analysis.

This analysis has several strengths. Although cancer drug shortages and stockouts are occurring at high frequency globally, it is relatively understudied in SSA. This analysis provides insight into the distribution of suboptimal therapy delivery events during stockout and non-stockout periods. Stockouts leading to switches and delays may account for some of the survival disparities that have been noted in this population, ${ }^{32}$ however, delays appear to occur despite no stockout, which warrants further studies to complement the current study.

As several African countries prepare to scale up cancer care, these data are generalisable to other countries on the continent where medication stockouts are also a problem, and breast cancer is the most common or second most common cancer diagnosed and may inform policies to guide selection of medicines to be included on national EMLs and also specific policies to ensure that medicines on the NEML are subsequently available at all times for patients for whom the drug is indicated. Countries need to implement policies that address chemotherapy procurement and supply chain. In a previous study, we outlined a detailed process for forecasting chemotherapy need within a universal healthcare coverage model to ensure a sustained supply of specific medicines on the NEML being used to treat prevalent cancers within the specific country context. ${ }^{33}$

\section{Author affiliations}

${ }^{1}$ Brigham and Women's Hospital, Boston, Massachusetts, USA

${ }^{2}$ Department of Radiation Oncology, University of Pennsylvania Perelman School of Medicine, Philadelphia, Pennsylvania, USA

${ }^{3}$ Department of Biostatistics, Epidemiology and Informatics, University of

Pennsylvania, Philadelphia, Pennsylvania, USA

${ }^{4}$ Oncology Department, Princess Marina Hospital, Gaborone, Botswana

${ }^{5}$ Department of Medicine (Infectious Diseases), University of Pennsylvania,

Philadelphia, Pennsylvania, USA

${ }^{6}$ Department of Medicine (Hematology-Oncology), University of Pennsylvania,

Philadelphia, Pennsylvania, USA

\section{Twitter Yehoda M Martei @ymartei}

Contributors Each author's contribution individually: SD: data analysis, interpretation of data, drafting the manuscript and approval of final version. SG: interpretation of data, revising drafts of manuscript and approval of final version. WBB: data analysis, interpretation of data, revising drafts of manuscript and approval of final version. DIS: interpretation of data, revising drafts of manuscript and approval of final version. TBR: interpretation of data, revising drafts of manuscript and approval of final version. PM: interpretation of data, revising drafts of manuscript and approval of final version. RG: Interpreted results, edited drafts of manuscript, approved final version. AD: interpretation of data, revising drafts of manuscript and approval of final version. LNS: interpretation of data, revising drafts of manuscript and approval of final version. YMM: conception, analysis, interpretation of data, drafting the manuscript, final approval of the version to be published.

Funding YMM's research work is supported by an NIH K01 Award K01TW011481, P30 Al 045008 NIH, Penn Center for AIDS Research Pilot Award and GMaP Region 4 Research Stimulus Award.

Competing interests SD, SG, DIS, TBR, PM, LNS and YMM have nothing to disclose. WBB reports personal fees from Genentech, outside the submitted work; Robert Gross reports grants from NIH, during the conduct of the study; personal fees from Pfizer, outside the submitted work; AD reports grants from Pfizer, grants from Novartis, grants from Genentech, grants from Calithera, outside the submitted work.

Patient and public involvement Patients and/or the public were not involved in the design, or conduct, or reporting, or dissemination plans of this research.

\section{Patient consent for publication Not required.}

Ethics approval Ethics approval was obtained from the University of Pennsylvania (Protocol \# 820159) and Botswana Ministry of Health (HRDC Reference \# HPDME 13/18/1). This was a retrospective study for which a waiver of consent was granted.

Provenance and peer review Not commissioned; externally peer reviewed.

Data availability statement Data are available on reasonable request, and will require local IRB approval or exemption in Botswana.

Open access This is an open access article distributed in accordance with the Creative Commons Attribution Non Commercial (CC BY-NC 4.0) license, which permits others to distribute, remix, adapt, build upon this work non-commercially, and license their derivative works on different terms, provided the original work is properly cited, appropriate credit is given, any changes made indicated, and the use is non-commercial. See: http://creativecommons.org/licenses/by-nc/4.0/.

\section{ORCID iDs}

Sonya Davey http://orcid.org/0000-0003-4907-9920

Yehoda M Martei http://orcid.org/0000-0002-5631-1567

\section{REFERENCES}

1 World Health Organization. Report of the WHO expert Committee, 2015 (including the 19th who model list of essential medicines and the 5th WHO model list of essential medicines for children). World Health Organization: the selection and use of essential medicines, 
2015. Available: http://apps.who.int/medicinedocs/documents/ s22190en/s22190en.pdf

2 Shulman LN, Wagner CM, Barr R, et al. Proposing essential medicines to treat cancer: methodologies, processes, and outcomes. J Clin Oncol 2016;34:69-75.

3 Robertson J, Barr R, Shulman LN, et al. Essential medicines for cancer: who recommendations and national priorities. Bull World Health Organ 2016;94:735-42.

4 Bazargani YT, de Boer A, Schellens JHM, et al. Selection of oncology medicines in low- and middle-income countries. Annals of Oncology 2014;25:270-6.

5 Martei YM, Chiyapo S, Grover S, et al. Availability of who essential medicines for cancer treatment in Botswana. $J$ Glob Oncol 2018;4:1-8.

6 Cherny NI, Sullivan R, Torode J, et al. ESMO International Consortium study on the availability, out-of-pocket costs and accessibility of antineoplastic medicines in countries outside of Europe. Ann Oncol 2017;28:2633-47.

7 World Health Organization. Meeting report: technical definitions of shortages and stockouts of medicines and vaccines. Essential Medicines and Health Products Department, 2016. https://www.who.

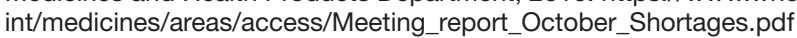

8 Havrilesky LJ, Garfield CF, Barnett JC, et al. Economic impact of paclitaxel shortage in patients with newly diagnosed ovarian cancer. Gynecol Oncol 2012;125:631-4.

9 Becker DJ, Talwar S, Levy BP, et al. Impact of oncology drug shortages on patient therapy: unplanned treatment changes. J Oncol Pract 2013:9:e122-8.

10 Nonzee NJ, Luu TH. The drug shortage crisis in the United States: impact on cancer pharmaceutical safety. Cancer Treat Res 2019;171:75-92.

11 Hedlund NG, Isgor Z, Zwanziger J, et al. Drug shortage impacts patient receipt of induction treatment. Health Serv Res 2018:53:5078-105.

12 Gupta S, Jain P, Gupta S, et al. 5-flourouracil (5FU) shortage: clinical implications and costs in a single month. J Glob Oncol 2012;30:663.

13 Metzger ML, Billett A, Link MP. The impact of drug shortages on children with cancer--the example of mechlorethamine. $N$ Engl J Med 2012;367:2461-3.

14 Poi MJ, Berger M, Lustberg M, et al. Docetaxel-Induced skin toxicities in breast cancer patients subsequent to paclitaxel shortage: a case series and literature review. Support Care Cancer 2013;21:2679-86.

15 Berger JL, Smith A, Zorn KK, et al. Outcomes analysis of an alternative formulation of pegylated liposomal doxorubicin in recurrent epithelial ovarian carcinoma during the drug shortage era Onco Targets Ther 2014;7:1409-13.

16 Martei YM, Binagwaho A, Shulman LN. Affordability of cancer drugs in sub-Saharan Africa: effects of pricing on needless loss of life. JAMA Oncol 2017;3:1301-2.

17 Martei YM, Grover S, Bilker WB, et al. Impact of essential medicine stock outs on cancer therapy delivery in a resource-limited setting. $J$ Glob Oncol 2019;5:1-11.

18 Lyman GH, Dale DC, Crawford J. Incidence and predictors of low dose-intensity in adjuvant breast cancer chemotherapy: a nationwide study of community practices. J Clin Oncol 2003;21:4524-31.
19 Lyman GH, Dale DC, Tomita D, et al. A retrospective evaluation of chemotherapy dose intensity and supportive care for earlystage breast cancer in a curative setting. Breast Cancer Res Treat 2013;139:863-72.

20 National Comprehensive Cancer Network. NCCN evidence-based cancer guidelines, oncology drug compendium, oncology continuing medical education, 2017. Available: https://www.nccn.org

21 Gianni L, Eiermann W, Semiglazov V, et al. Neoadjuvant and adjuvant trastuzumab in patients with HER2-positive locally advanced breast cancer (NOAH): follow-up of a randomised controlled superiority trial with a parallel HER2-negative cohort. Lancet Oncol 2014;15:640-7.

22 Gianni L, Dafni U, Gelber RD, et al. Treatment with trastuzumab for 1 year after adjuvant chemotherapy in patients with HER2-positive early breast cancer: a 4-year follow-up of a randomised controlled trial. Lancet Oncol 2011;12:236-44.

23 Willson ML, Burke L, Ferguson T, et al. Taxanes for adjuvant treatment of early breast cancer. Cochrane Database Syst Rev 2019;9:CD004421.

24 Management Sciences for Health (MSH). International medical products price guide, 2016. https://www.msh.org/resources/ international-medical-products-price-guide

25 Schmoll H-J, Twelves C, Sun W, et al. Effect of adjuvant capecitabine or fluorouracil, with or without oxaliplatin, on survival outcomes in stage III colon cancer and the effect of oxaliplatin on post-relapse survival: a pooled analysis of individual patient data from four randomised controlled trials. Lancet Oncol 2014;15:1481-92.

26 Nakanishi M, Kuriu Y, Murayama Y, et al. A study on the tolerability of capecitabine plus oxaliplatin as adjuvant chemotherapy. Anticancer Res 2016;36:1851-4.

27 Denduluri N, Patt DA, Wang Y, et al. Dose delays, dose reductions, and relative dose intensity in patients with cancer who received adjuvant or neoadjuvant chemotherapy in community oncology practices. J Natl Compr Canc Netw 2015;13:1383-93.

28 NCCN. Recently updated NCCN clinical practice guidelines in OncologyTM, 2020. Available: https://www.nccn.org/professionals/ physician_gls/recently_updated.aspx

29 Aapro MS, Cameron DA, Pettengell R, et al. EORTC guidelines for the use of granulocyte-colony stimulating factor to reduce the incidence of chemotherapy-induced febrile neutropenia in adult patients with lymphomas and solid tumours. Eur $J$ Cancer 2006;42:2433-53.

30 O'Neil DS, Chen WC, Ayeni O. Breast cancer care quality in South Africa's public health system: an evaluation Using American Society of Clinical Oncology/National Quality Forum Measures. J Glob Oncol 2019;5.

31 McBride A, Holle LM, Westendorf C, et al. National survey on the effect of oncology drug shortages on cancer care. Am J Health Syst Pharm 2013;70:609-17.

32 Reeder-Hayes KE, Anderson BO. Breast cancer disparities at home and abroad: a review of the challenges and opportunities for systemlevel change. Clin Cancer Res 2017;23:2655-64.

33 Martei YM, Chiyapo S, Grover S, et al. Methodology to forecast volume and cost of cancer drugs in low- and middle-income countries. J Glob Oncol 2018;4:1-8. 